Research Article

\title{
Urban Planning Image Feature Enhancement and Simulation Based on Partial Differential Equation Method
}

\author{
Duo Li (iD \\ Department of Architecture, Nanyang Institute of Technology, Nanyang, Henan 473004, China \\ Correspondence should be addressed to Duo Li; 3051003@nyist.edu.cn
}

Received 28 July 2021; Revised 12 August 2021; Accepted 14 August 2021; Published 2 September 2021

Academic Editor: Miaochao Chen

Copyright (c) 2021 Duo Li. This is an open access article distributed under the Creative Commons Attribution License, which permits unrestricted use, distribution, and reproduction in any medium, provided the original work is properly cited.

\begin{abstract}
Based on the introduction of the basic ideas and related technologies of partial differential equations, as well as the method of path planning, the application of partial differential equations in solving urban path planning is studied. The path planning model of partial differential equations and the setting of obstacle boundary conditions are introduced, and adaptive. Theoretical research and experimental results show that it is feasible and effective to solve urban path planning by partial differential equations, which provides a new way for urban path planning research ideas and methods. This paper proposes an image detection algorithm based on diffusion equation. According to the logarithmic transformation, the multiplicative speckle noise in the image can be converted into additive noise. We first perform logarithmic transformation on the image and then use the denoising model of the diffusion equation to filter out the noise in the image and then use the logarithm to recognize the image. The difference image is obtained by the domain difference method, and finally, the difference image is classified by the clustering algorithm, and the change area is extracted. Experiments show that the algorithm can effectively reduce the effect of multiplicative speckle noise on the change detection results, improve the accuracy of change detection, and shorten the change detection time. This article takes the path planning problem of a two-dimensional space city as an example to discuss the application of partial differential equations. According to the principle of energy conservation, this paper uses the two-dimensional space radiant heat conduction equation as an example to model and illustrate the solution of the path planning problem.
\end{abstract}

\section{Introduction}

With the development of human society, the society has higher and higher technical requirements for multimedia information processing. Images are an important source for humans to obtain external information. Digital image processing technology has become a research hotspot in the field [1]. Under normal circumstances, the process of digital image processing will be interfered by different noise signals, which will reduce the quality of the image. This not only makes the image blurry but also affects the subsequent processing of the image, such as image segmentation and image compression [2]. Therefore, finding a fast and effective image denoising algorithm is a big challenge facing researchers. Image restoration has always been a concern in the field of image processing, and it is also a preprocessing process for high-level image processing applications [3]. Traditional image restoration methods often destroy image features such as edges, lines, and textures while removing noise. Algorithms based on partial differential equations can maintain the details of the image while restoring the image. Therefore, it has received more and more attention in recent years [4]. Urban path planning is an important link and subject of navigation research. Traditional path planning methods mainly include artificial potential field method, genetic algorithm, artificial neural network, simulated annealing algorithm, ant colony optimization algorithm, and RRT random search algorithm [5]. The artificial potential field algorithm is the most typical algorithm. It has mathematical completeness and simplicity, but it is difficult to deal with obstacles and narrow channel oscillations. The advantage of intelligent algorithms such as genetic algorithm and ant colony algorithm is that the path can converge to the optimal or it is approximately optimal. The disadvantage is that the evolution 
speed is difficult to control, and too many empirical parameters are required, which is not conducive to automatic processing [6]. The RRT algorithm is a single query path planning method based on sampling, because it avoids the modeling of the space. Compared with the method, it has unique advantages, but because the random search is uniform in the global space, the algorithm is meaninglessly expensive and the planning time is longer [7].

Partial differential equations can effectively simulate dynamic processes, such as one-dimensional string vibration, two-dimensional membrane vibration and heat conduction, and three-dimensional acoustic wave oscillation. Therefore, they are widely used in physics, mechanics, engineering technology, and other natural science fields [8]. The rich content and better performance of partial differential equations provide new enlightenment for urban path planning algorithms. Image restoration is also called image restoration; it is the process of restoring degraded images. It can be seen that image restoration is actually the inverse process of the image degradation process [9]. The purpose of image restoration technology is to restore the degraded image to its original appearance as much as possible. The method is to first analyze the mechanism of image degradation, that is, use mathematical models to describe the process of image degradation. Then, on the basis of the degradation model, through the master's thesis based on the partial differential equations of the image restoration research to find the mode calculation of the inverse process, the real image can be obtained more accurately from the degraded image, and the original information of the image can be restored [10]. Image restoration has always been a concern in the field of image processing, and it is also a preprocessing process for high-level image processing applications. Image restoration occupies an extremely important position in primary visual processing and has a wide range of applications in aerospace, national defense, public security, biomedicine, cultural relic restoration, and other fields [11].

This paper introduces the path planning problem of partial differential equation modeling and proposes a new urban path planning algorithm. At the same time, an image change detection based on an adaptive total variation (TV) denoising algorithm is proposed. First, the image is converted to the logarithmic transform domain, and then, the image is denoised using the adaptive total variation denoising model. In this algorithm, in view of the shortcomings of the original TV denoising algorithm, we use a generalized energy function and introduce a difference variable to automatically select the number of iterations required for denoising, avoiding insufficient denoising or waste caused by manual selection of the number of iterations matter of time. Finally, the $\mathrm{k}$-means clustering algorithm is used to cluster the difference images. (1) Considering the step effect of P-M in the process of restoring images, a complex diffusion model that couples median filtering and the P-M model is improved. Without affecting the denoising performance, the visual effect of the image is improved. (2) Considering that the model cannot effectively remove the shot noise points, it is improved, and a time-weighted selective diffusion model is proposed. (3) Considering that the total variation model will have a step effect in the process of restoring the image, an improved model that couples the second-order and fourth-order partial differential equations is proposed. The improved algorithm improves the visual effect of the image, and the convergence speed is much higher than that of the fourthorder model. Experimental results show that the adaptive total variation denoising algorithm can effectively suppress image noise and improve the detection accuracy and efficiency of the algorithm. This paper focuses on the research of image modeling and studies three different image restoration models from different perspectives for the basic problems of image restoration. The first type of model is a restoration model based on the scale-space axiom system. The main research is to analyze and design anisotropic diffusion equations from the perspective of directional filters; the second type of model is partial differential equation models derived from the perspective of functional variation, focusing on the total variation models and four that are widely used today. The third type of model is a hyperbolic partial differential equation model called impulse filtering, which can be used for image enhancement or deblurring.

\section{Related Work}

Torquato and Haslach Jr. [12] use hyperbolic partial differential equations to simulate the path of the endoscope in the human body. The advantage is that it can handle the peek path of various organ structures, and the propagation speed in one direction depends on its investigation solution. This is limited. The solution may be singular within time, leading to the rupture of the solution. According to the order in which the images are classified in the change detection process, the image change detection method can be divided into two types: comparison before classification and comparison after classification. The method is to first classify the two images separately and then compare the two remote sensing images after classification. This type of algorithm relies on the accuracy of the classifier, and the initial error will accumulate in the subsequent comparison process.

The first comparison and then classification method generally obtains the difference image first and then classifies the difference image to obtain the detection result. The accuracy of such image change detection mainly depends on the quality of the difference image and the accuracy of the difference image classification algorithm. For example, Naghavi et al. [13] proposed multiscale and multitemporal satellite image change detection (MCD algorithm for short), which uses logarithmic ratio algorithm to calculate the difference image of SAR image, uses undecimated discrete wavelet to decompose the difference image, and finally uses k-means clustering algorithm that clusters the decomposed difference images to obtain the detection results. Evans [14] and others proposed SAR image change detection based on difference image fusion and $\mathrm{k}$-means clustering (PPK algorithm for short), which uses PPB Filter denoising; they use weighting method to combine logarithmic ratio difference images and difference images in the time domain and then use $\mathrm{k}$ means clustering algorithm to cluster the difference images, 
which improves the accuracy of change detection, but the detection time is relatively long. Berger and Oliger [15] and others proposed a SAR image change detection based on the fusion of ratio image data and fuzzy clustering (CIVB algorithm for short). The algorithm uses a contextindependent variable fusion algorithm to fuse the mean ratio difference image and the log ratio difference image and then uses fuzzy clustering algorithm to process the difference images. This algorithm improves the effect of change detection, but after obtaining the fused difference image, it needs to use multiple scales to extract homogeneous regions, and the total running time is very long. Others proposed that all image change detection can be classified according to the detection purpose, detection data, detection dimension, detection time scale, and detection content and divided the image change detection technology into direct comparison method, classification comparison method, object-oriented method, and model seven methods, such as method, time trajectory method, visualization method, and hybrid method.

In recent decades, image denoising processing technology based on partial differential equations has become a research hotspot in the field of image processing, and it has gradually taken shape with the introduction of image smoothing and multiscale space theory. Later, Pai [16] first gave a model based on anisotropic diffusion, which is called the Perona-Malik model (PM model), which overcomes the disadvantages of isotropic diffusion. The biggest advantage of the PM model is to combine the smoothing process of the image with edge detection, use the edge spread function to control the spread rate of the edge, and try to spread in the nonedge area of the image, so this model can not only effectively remove noise but also, moreover, the edge texture of the image can be sharpened, which lays the foundation stone for the further development of digital image processing technology. However, the model still has defects. There is uncertainty in the choice of the model's diffusion coefficient. In addition, it is easy to ignore the strong isolated noise and edges in the image, which will cause a serious step effect. In order to overcome these shortcomings, later researchers have proposed many improved algorithms. Huo and Chai [17] proposed an image denoising model based on the curvature of a weighted Gaussian function. Helbing et al. [18] proposed an algorithm based on anisotropic diffusion, mainly for speckle noise, using the probability density function of the edge and the correlation information of the pixel to control the diffusion rate, which not only protects the edge information but also solves the image oversmoothing defects. Some scholars have proposed a modified diffusion model based on adaptive edge threshold, which improves the PM model and mainly introduces a directional filter [19]. This coefficient takes into account the variability of local pixels in order to better remove edge noise and selectively smooth the image. Some scholars introduced the directional Laplacian operator to improve the PM model, which diffuses the image along the edge of the original image, effectively alleviating the step effect, and protecting the sharp edges. This model can effectively remove noise while preserving edges, borders, and textures [20, 21].

\section{Urban Planning Image Feature Enhancement Model Construction Based on Partial Differential Equation Method}

3.1. Method Hierarchy of Partial Differential Equations. Given an equation, generally, it can only describe the general law of a certain motion, and the specific state of motion cannot be determined, so the equation is called a universal equation. If some conditions are attached (such as the condition of the start of motion or the boundary after being subject to external constraints), the specific motion state can be completely determined, and such conditions are called definite solution conditions. The additional conditions that represent the starting situation are called initial conditions, and the conditions that are restricted on the boundary are called boundary conditions. Figure 1 shows the partial differential equation method hierarchical distribution structure.

Suppose that the function $x(t)$ satisfies the universal definite equation in the region $R$. When the point tends to give the initial hyperplane or the boundary surface of the boundary condition from the region $R$, the $u$ required in the definite solution condition and its limit of the derivative exists everywhere and satisfies the corresponding definite solution condition, so $x$ is called the solution of the definite solution problem.

$$
X[t]=\{x \in R \mid x(i), t \in R\}, \quad i=1, \cdots, t .
$$

The constrained least power recovery is derived based on function smoothing. This restoration method establishes an optimal criterion based on a smoothness measure, such as minimizing the second derivative of the image (i.e., Laplacian operator). In order to be meaningful, the recovery process must be constrained with known parameters. Therefore, it is necessary to find the minimum value of the criterion function $u(x, t)$. For example, the function $u(x, t)$ can be defined as

$$
u(x, t)=\left[\begin{array}{ccc}
x(1) & 0 & 0 \\
0 & \cdots & 0 \\
0 & 0 & x(t)
\end{array}\right]\left[\begin{array}{ccc}
0 & 1 & 1 \\
1 & \cdots & 1 \\
1 & 1 & 0
\end{array}\right]
$$

If all the conditions are met (the existence of the filter function and the existence of the inverse Fourier transform), for the restored signal $x$, force is equal to the ideal signal $f$ $(x, t)$, so there is a perfect restoration. The edge gradient of the image is large, the coefficient is small, the diffusion is weak, and the edge is maintained; the internal gradient of the image is small, the diffusion coefficient is large, the equation is close to the heat conduction equation, the diffusion strength is large, and the image is smoothed, thus realizing the anisotropic diffusion ability. It is obtained by passing an imperfect image through a linear filter. Logically, the filter $w$ is called an inverse filter, and its transfer function is the inverse of the transfer function of the degraded $\operatorname{div}(u)$ : 


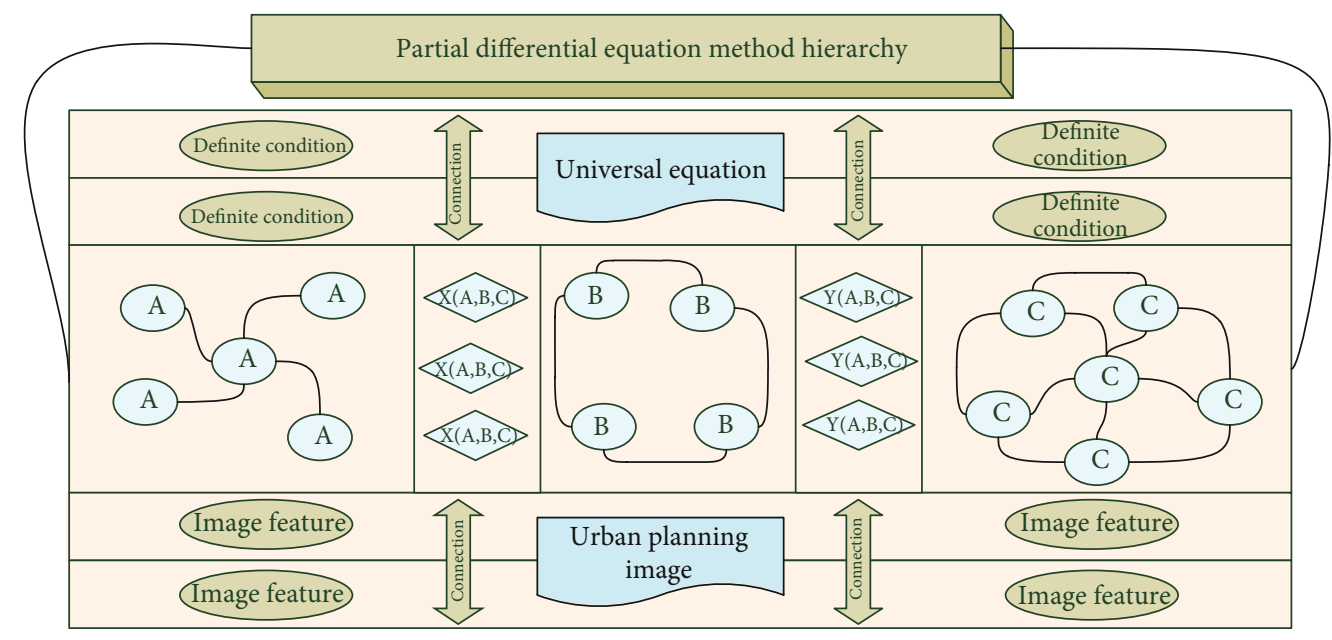

Figure 1: Partial differential equation method hierarchical distribution structure.

$$
\begin{gathered}
\frac{\partial u(x, t)}{\partial t}=\operatorname{div}(c(x, t) \times \nabla u), \quad x, t \in R \\
f(x, t)=\operatorname{div}(|\nabla u| \nabla u)+\nabla u(\nabla x(t)) \nabla t
\end{gathered}
$$

The motion law of many objects can be described by wave equations. For example, string vibration can be described by one-dimensional wave equation; membrane vibration can be described by two-dimensional wave equation; acoustic and electromagnetic wave oscillations can be described by threedimensional wave equation. Therefore, the path planning of mobile robots can be used with the two-dimensional or three-dimensional equation description of the vibration or wave from the start point to the end point.

$$
\begin{gathered}
\min u(x, t \mid t \in(0, \infty))=\frac{\operatorname{conv}(u(x)-u(0), u(x))}{\sqrt{\operatorname{var}(u(x)-u(0)) \cdot \operatorname{var}(u(x))}}, \\
g(|\nabla u|)-e^{-|\nabla u| / k}=0, \\
\operatorname{conv}(X, Y)-E[(X-\bar{X})(Y-\bar{Y})]=0 .
\end{gathered}
$$

If this condition is met, the mold can be mutually used iteratively. It needs to be pointed out that because all operations are a combination of image information, no other features are added in this process, so the larger the scale parameter $v(x, t)$ is, the simpler the content of the image is.

$$
\begin{aligned}
& |\nabla u| \operatorname{div}(\nabla u)=\frac{u(x) x(t)-2 u(x)^{2}}{\nabla u(x) \nabla x(t)+u(x) x(t)}, \\
& \left\{\begin{array}{l}
v(x, t)-\frac{k^{2}}{k^{2}+|\nabla u|^{2}}=0, \\
v(x, 0)=v(t) x(0) .
\end{array}\right.
\end{aligned}
$$

It can be seen from the above formula that if $\operatorname{div}(u, v)$ takes 0 or is very small on the plane, it will bring about computational difficulties. On the other hand, noise can cause more serious problems. In this case, recovery can only be performed within a range close to the origin (close to the center of the frequency domain).

3.2. Image Feature Enhancement Algorithm. The physical domain $g$ is the passable area of $u(x, y)$ with the city where the obstacle is removed in the working environment. If the obstacle is dynamic, the obstacle is divided according to the size and direction of the speed. The boundary is set to the corresponding multiple relationships of the Dirichlet condition.

$$
\begin{gathered}
\sum_{i=1}^{n} g(|\nabla u \cdot x(t)|, u(x), u(y)) \cdot \sqrt{\frac{1}{(1+\nabla u(x) \nabla x(t))^{2}}}=-1, \\
\frac{\partial u(x, y, t)}{\partial x(t)}+\frac{\partial u(x, y, t)}{\partial y(t)}+\frac{\partial u(x, y, t)}{\partial y(x)}=0 .
\end{gathered}
$$

Figure 2 shows the flow chart of the image feature enhancement algorithm. Therefore, the algorithm for solving the model equation based on the adaptive grid is as follows: (1) initialize the city's passable area as a quasiregular triangulation grid, set $u$ as the preset uniform grid unit side length value, and set $t$ as the passable area of the minimum value of the energy preset; (2) use the finite element difference format to obtain the approximate solution of $y(t)$ on the triangulation grid of the city starting area in $R$.

$$
\begin{gathered}
\frac{\nabla u(x, y, t)}{\nabla t}-\frac{u(x+t, y)-u(x, y)}{t+1}=0, \\
{[x(i) \cdots x(1)]\left[\begin{array}{c}
y(1) \\
\cdots \\
y(i)
\end{array}\right]=\frac{\partial u(x, y, t)}{\partial u(t)} \cdot \nabla u .}
\end{gathered}
$$

If the error meets the predetermined tolerance, the grid accuracy is normal. We perform linear interpolation between the triangle node and the midpoint and return the gradient of 


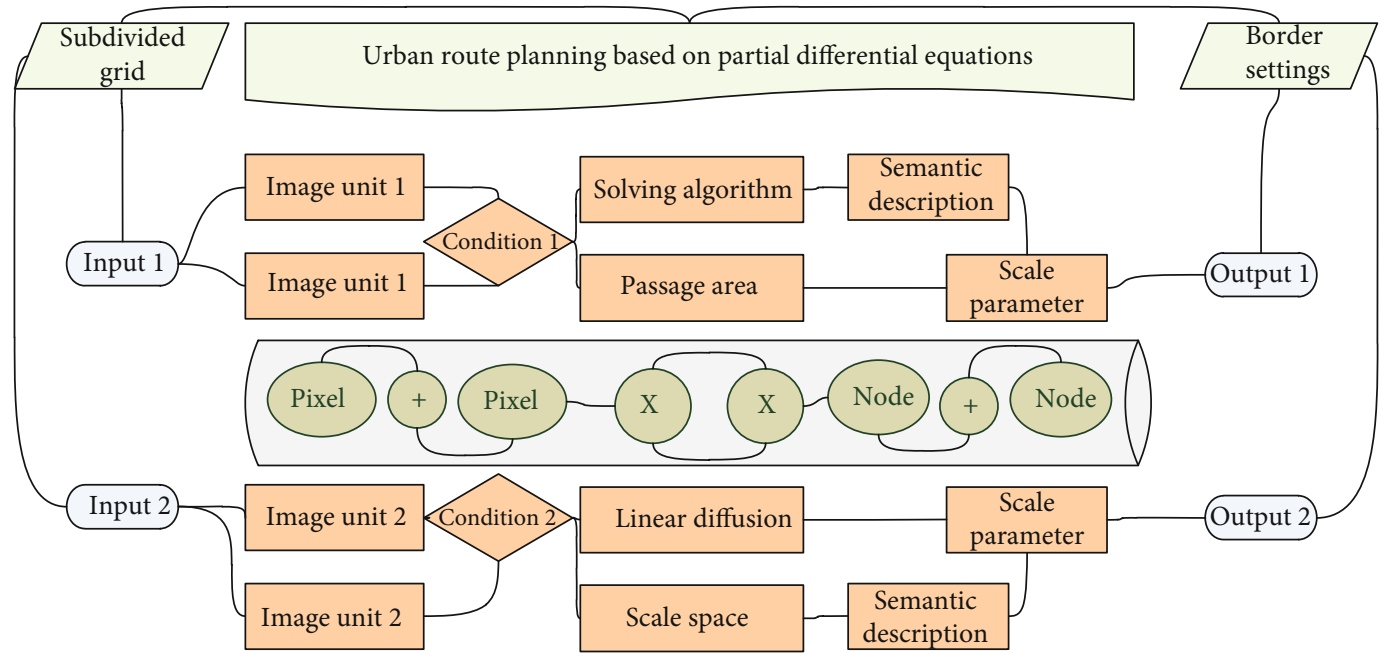

FIgURE 2: Flow chart of image feature enhancement algorithm.

the triangle center solution. If the gradient is less than the preset energy minimum, the calculation ends; otherwise, it will enter the next step of grid and gradient calculation.

$$
\begin{gathered}
\Delta u(x, t)=\sum_{i=1}^{n} \frac{\nabla u(s, t)}{\nabla u(x)}=\frac{u(s+t, t)-u(x, t)}{x(s, t)}, \\
\left\{\begin{array}{l}
\frac{\Delta u(x, t)}{u(x, t)}=\frac{\partial u(x, y, t)}{\partial u(t)} * \operatorname{div}(c(\operatorname{Im}(u(x, t)) \Delta u(x, t))), \\
\frac{\Delta u(x, 0)}{u(x, 0)}=\partial u(x, 0) .
\end{array}\right.
\end{gathered}
$$

Linear diffusion is the oldest and best way to understand scale space. From the perspective of scale space, the image after the continuous family of operators will get a series of smoother images. The original image corresponds to the scale $t$. For the image at $=0$, an image with a simpler structure can be obtained by increasing the scale t. Because the scale-space theory introduces a hierarchical structure in the image characteristics, it constitutes an important step in the transformation from pixel-related image expression to semantically described image expression.

For a fixed node $x$, let $y$ traverse all the nodes adjacent to $x$ in the grid, and the redistributed nodes after the refinement form a new grid. The default is triangle division, and all nodes are the vertices of the triangle. After adding a new adaptive grid step by step, return to step to recalculate. Research shows that more than $70 \%$ of all information received by humans is obtained through vision. Compared with voice and text information, images contain larger, more intuitive, and more accurate information and therefore have higher practical efficiency and wide applicability. When images are processed and transmitted in digital form, this storage and transmission format has become the main development trend in this field due to its advantages such as good quality, low cost, miniaturization, and easy implementation.
However, in the process of image formation, recording, processing, and transmission, due to the imperfect imaging system, recording equipment, transmission medium, and processing method, the image quality may be reduced, which cannot fully reflect the real content of the scene. This phenomenon is called image degradation. In this model, the image degradation process is modeled as a system function that acts on the input image (usually called the point spread function). It is combined with an additive noise $n(x)$, which forces to produce a degraded image $g(x, y)$. The concept of physics is introduced into the image, and the whole image can be regarded as a flat plate with different heat. The heat conduction equation follows that time changes make the temperature of the entire plate tend to balance. The hightemperature point delivers energy to the low-temperature point to cool down, and the low-temperature point accepts energy to heat up until the iteration is terminated. The noise points in the image can be regarded as high-temperature energy points, which follow the equation evolution; the frequency gradually decreases until it is smoothed out.

3.3. Optimization of Planning Evaluation Factors. In the process of smoothing the image with the Perona-Malik equation, sometimes "blocking effect" appears. That is, the grayscales in some areas after the 4 image processing are the same. The same grayscale in the area means that the first-order reciprocal of the gray value at any point in the area is 0 . This shows that as the number of iterations increases, the image transitions to the same grayscale image. Figure 3 shows the comparison of the histogram of image feature smoothness. This can also be seen from the assumption of the image model in the robustness estimation method. In the robustness estimation method, the assumed image is the sum of the horizontal image of the block (the gradient of each point in the same block is zero) and the noise with zero mean and small variance. Using an attenuated enhancement factor can make high-contrast pixels less enhanced, and the enhanced image result is generally softer, and the visual effect is not too sharp, but some useful but 


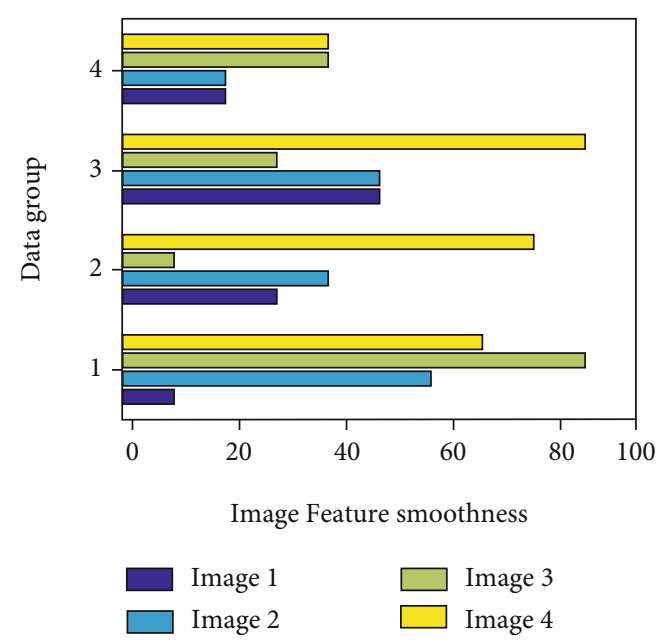

FIGURE 3: Comparison of smoothness histograms of image features.

originally difficult to observe details are better enhanced. In this way, smoothing and eliminating noise multiple times will inevitably make the image transition to a block-level image. If we change $u$ to the form of $v$ ( $v$ is the Laplacian operator), when $v=0$, the image is only a plane, but a horizontal plane is not necessarily required.

In order to avoid damage to image features and achieve good results, smoothing should follow the following two principles: (1) the areas with strong image features are less smooth; the areas with weak image features are more smooth; (2) the vertical smoothness of the image feature is small; the smoothness along the image feature is large. In the direction of image features which are mentioned, the method based on partial differential equations judges the flatness of the image according to the size of the gradient during processing. This can be seen from the pixel point gradient in the coefficient distribution function during iteration. On the other hand, it also detects the direction of the image feature and reduces the smoothness in the direction perpendicular to the feature. Accurately extracting the image feature direction is critical for image smoothing and other image processing tasks. For some image feature directions, gradient-based extraction methods are prone to errors. Partial differential equations began to be used in physics to describe the objective world. Later, after continuous in-depth research by researchers, partial differential equations have gradually been applied to the field of digital image processing, and good results have been achieved in this field. The image denoising method of differential equation not only effectively filters out the noise in the image but also the important structural feature information of the image is basically not lost. In addition, the image denoising algorithm based on partial differential equations is a nonlinear method and has a relatively complete mathematical theory system and design structure. In addition, the calculation is fast, simple, accurate, and stable. More importantly, the partial differential equations are closely related to the physical characteristics of the image such as gradient and variance.

\section{Application and Analysis of Urban Planning Image Feature Enhancement Model Based on Partial Differential Equation Method}

4.1. Numerical Processing of Partial Differential Equations. The image itself has a complicated structure, the pixel gray value of the noise and texture detail area of the image is too large, and the gray value of the flat area is relatively small. Taking into account this characteristic, the frequency domain low-pass filter is used to filter out the noise signal, and then, the image information of the low-frequency part is retained, so as to achieve the purpose of removing noise. Partial differential equations use the same diffusion strategy in different regions, that is, regardless of the direction, the diffusion capacity and speed of the equation are the same. The ideal conductivity coefficient should be more spread in the smooth area, less spread around the grayscale changes, so as to protect the edge while smoothing out some small changes such as noise and some unnecessary textures. However, the image itself has complex structural information, and the use of the same diffusion coefficient will inevitably ignore the importance of the image's structural feature information, which in turn leads to image distortion. The model uses the local gradient characteristics of the image to adaptively determine the diffusion coefficient. Different regions and moments have different diffusion coefficients, which can effectively retain the edge information and texture details of the image. Figure 4 shows the denoising effect curve distribution at different noise points in the image. Specifically, in areas where noise is densely distributed, the diffusion ability is relatively large, which can filter out the noise points of the image; in the edge area of the image, the spread function is almost no longer diffused, thereby protecting the edge of the image from being affected by the filter.

In order to verify the effectiveness of the method in this paper, the article selects a $128 \times 128$ image as the test image and adds various mixed noises to it (this article takes one of the mixed noises as an example, the Gaussian noise and density with a variance of $0.01 \%, 0.05 \%$ of the salt and pepper noise is mixed), and then, the second-order PDE denoising model, the fourth-order YK model, the LC model, and the model in this paper are used for denoising comparison. According to the classification accuracy effect on the database, the results are divided into two groups for analysis, as shown in the paper.

It can be seen from the classification results that the classification accuracy of the method is greatly improved compared to other methods. Figure 5 shows a schematic diagram of the numerical processing effect of partial differential equations. The scene contains obvious foreground targets and background with similar local structure. The classification effect of low-rank coding methods such as LrrSPM(Fast Low-rank Representation based Spatial Pyramid Matching) is significantly higher than that of sparse coding ScSPM, because these images have similar or uniform structural features. The low-rank coding adopts a joint coding method, and the minimum rank regularization can capture these features. Commonality at the same time, it can also be seen that the Local Coding Method (LLC) is better than the Sparse 


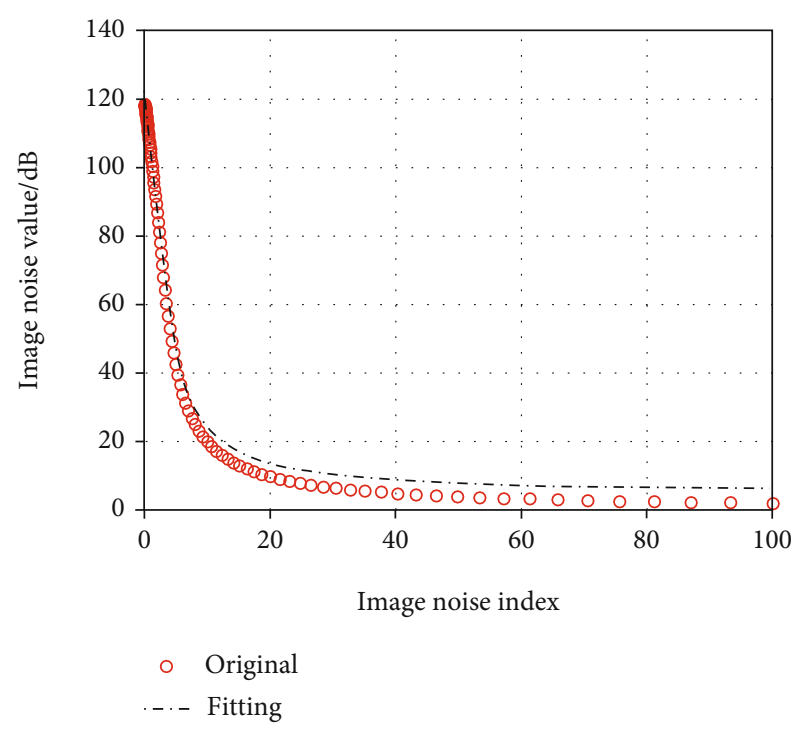

Figure 4: Denoising effect curve distribution at different noise points of the image.

Coding (ScSPM). The reason for the analysis is that the local features of each type of image contained in the scene are extremely similar; the local features of the same snowboarding are very similar. It is further verified that local constraints can effectively capture the similarities and common features between local feature descriptors.

4.2. Image Feature Model Simulation. The experimental environment is developed with Matlab, running on a PC, CPU frequency is $512 \mathrm{MB}$, and collision radius is $0.4 \mathrm{~m}$. The environment is a rectangular area under $20 \mathrm{~m} \times 20 \mathrm{~m}$, obstacles are randomly set, and the size is arbitrary. Dynamic environment parameters are random settings. The starting point is set to $(0,100)$, and the end point is set to $(100,0)$. When there are dynamic obstacles in the experimental environment, we use the heat conduction partial differential equation to solve the urban path planning. In the obstacle, point 1 is the starting point of movement, point 2 initially meets the city, point 3 meets the boundary of the solid wall, and point 4 is the path point the ball has traveled through. It can be seen that the adiabatic condition of the obstacle boundary affects the heat conduction in the planning process, making the original heat conduction direction change. By predicting the movement speed of obstacles, the obstacle avoidance and detour can be successfully achieved. Because the movement speed of obstacle is too large, it does not affect the original conduction planning path, so there is no need to detour.

In order to analyze the effectiveness of the proposed adaptive fractional denoising algorithm based on the improved PM model, four grayscale images with a size of $512 \times 512$ are selected for comparative analysis. Secondly, combined with the experimental results, we use Matlab to simulate the experiment. Solving the lowest matrix representation of a 128-dimensional image gets a rank of 59, which shows that the rank of natural images is much smaller than the dimensionality, it is divided into several small blocks according to the local similarity of the image, and the minimum matrix representation of each local small block is obtained. It can be seen that local constraint coding can capture the relationship of similar features. Figure 6 shows the image threshold parameter gradient denoising effect distribution. There is also an important parameter $K$ in the proposed model. The value of the threshold parameter $K$ determines the gradient value that starts to weaken the diffusion ability. If its value is unreasonable or inaccurate, there will be incomplete noise filtering or excessively smooth flat areas and the loss of edge information.

Figure 7 shows the before and after comparison of the convergence speed of the image algorithm. In order to see the influence of $K$ value on the diffusion rate more intuitively, here, we take $K$ as 20 and 100, respectively, to draw a graph of the relationship between diffusion coefficients and gradient modulus. The image signal-to-noise ratio after denoising is 11.83 , and the calculation time is 21.44 seconds. The noise in the image has been removed to a certain extent, but the visual effect is still not satisfactory, and the convergence speed of the algorithm is too slow, resulting in the model's denoising running time is too long. For the parameters, we improved their values. In the paper, we set the parameter $s=70$, the quantity $=20$ (the value of the harmful value in Matlab is the minimum real number), the visual effect of the image after denoising is better, and the visual quality of the image has been improved to a large extent. The image SNR after denoising is 14.37, and the calculation time is greatly shortened to 19.66 seconds.

4.3. Example Application and Analysis. It is the planned path obtained after solving the elliptic partial differential equation mobile robot path planning model established in this paper. Due to the adaptive mesh refinement of the experimental environment, the planned path remains in a narrow area. It can be passed safely, and the planned path is relatively smooth, avoiding sudden changes in city-related operating parameters, so it can be applied to path planning in incomplete cities. All experiments in Matlab R2012a for Windows 10 are used for simulation, and computer configuration is an Intel Core i5 $2.40 \mathrm{GHz}$ and a $4 \mathrm{G}$ operating core. From the paper, the setting of obstacle boundary conditions and the three-dimensional results of model calculation can be seen more intuitively with the results of smoothing experiments on images with fluctuation characteristics.

Figure 8 shows the box plot of the denoising image recognition degree deviation. It can be seen that when image pixels are used as the diffusion function, the denoised image has a better recognition effect. Therefore, it shows that the two diffusion coefficients can effectively remove the noise. However, according to the data, the PSNR (peak signal-tonoise ratio), SSIM (Structural Similarity), and information entropy of the denoised image when image index 1 is used as the spread function are slightly higher than the corresponding values of the denoised image when image index 2 is used as the spread function. In addition, when image index 1 is used as the spread function, the running time of the denoising image is significantly shortened. Therefore, 


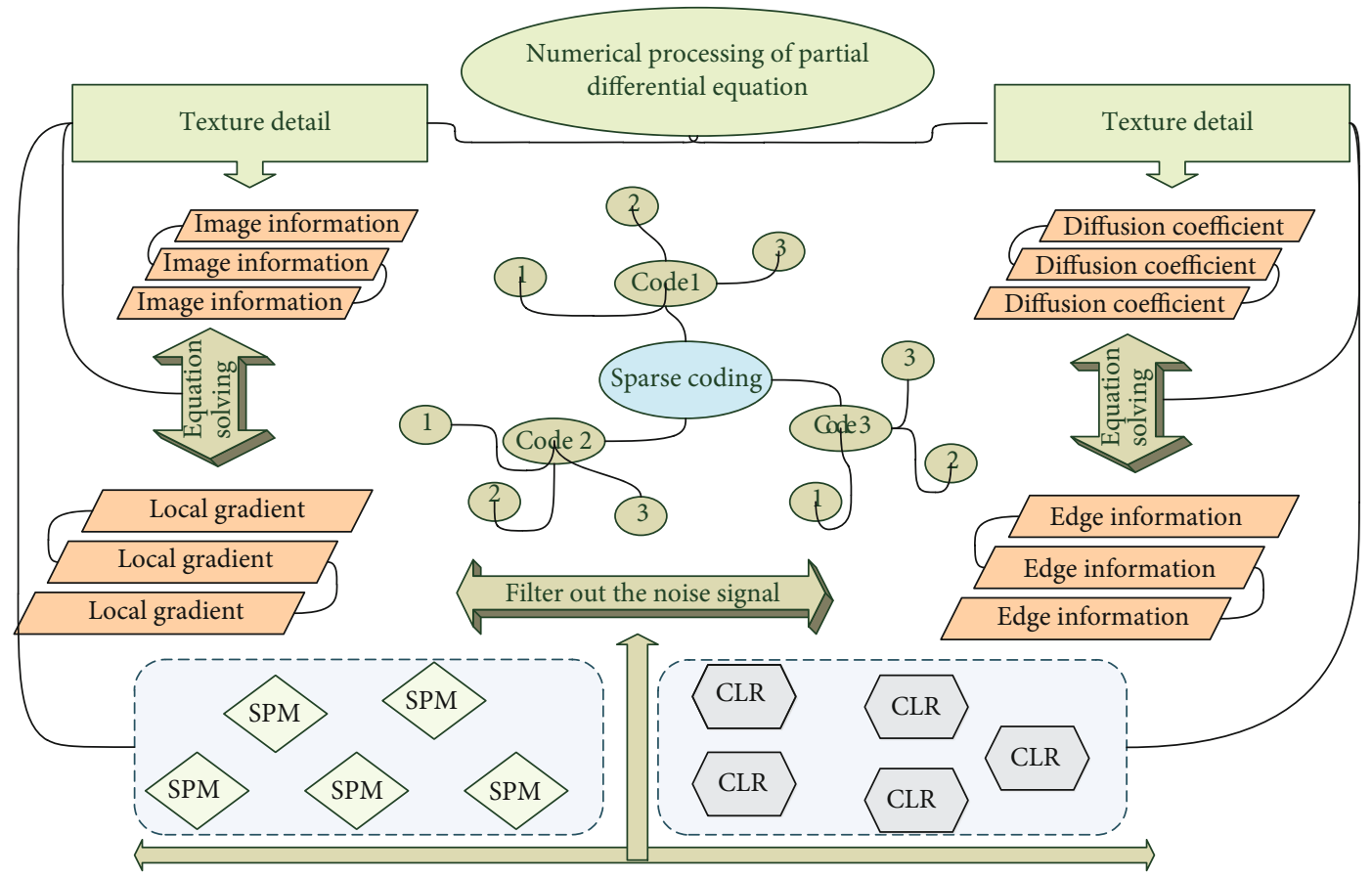

FIGURE 5: Schematic diagram of the numerical processing effect of partial differential equations.

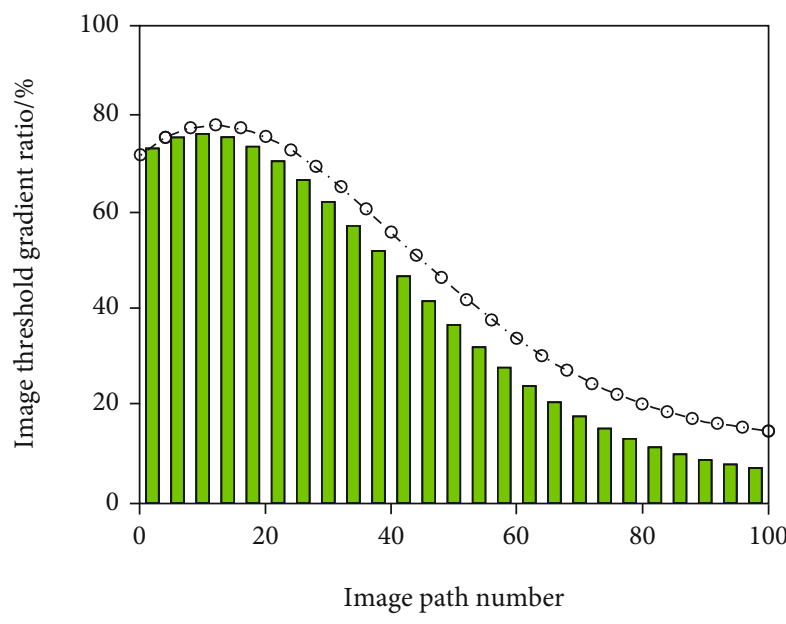

Figure 6: Image threshold parameter gradient denoising effect distribution.

the spread function image index 1 is selected to analyze the image in the following models.

Figure 9 shows a three-dimensional histogram of the peak signal-to-noise ratio versus pixel diffusion time. It can be seen that the peak signal-to-noise ratio value gradually decreases as the number of diffusion increases, because the larger the peak signal-to-noise ratio value is, the longer the diffusion time and the loss of image texture details are, so the peak signal-to-noise ratio value should theoretically conform to the decreasing function. The basic idea is to gradually increase the number of diffusions until the peak signal-to-noise ratio reaches the maximum after the diffusion starts. After each iteration of the noise image, the noise

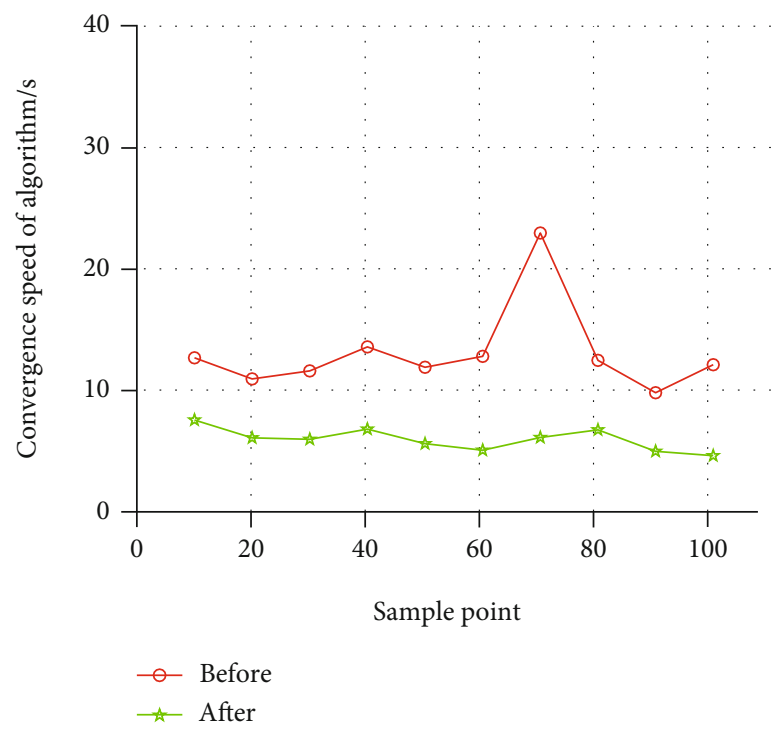

FIGURE 7: Convergence speed of image algorithm before and after comparison line chart.

image will become smoother. The smoother the image is, the smaller the gradient of the image is. It can also be seen from the results that the effect of sparse coding is better than that of low-rank coding, which shows that sparse coding adopts an independent coding method for each image to preserve the individual characteristics of the image, but this coding method has differences in characteristics. If a slightly changed image is added to a similar scene class, the coding coefficient will change greatly. Therefore, as the number of diffusion increases, the value of peak signal-to-noise ratio 


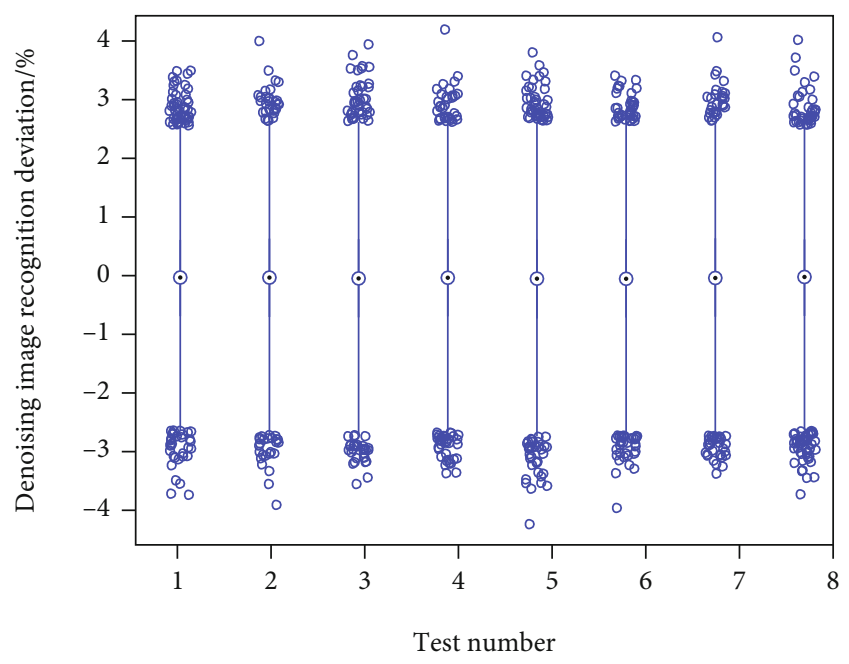

Figure 8: Box diagram of denoising image recognition degree deviation.

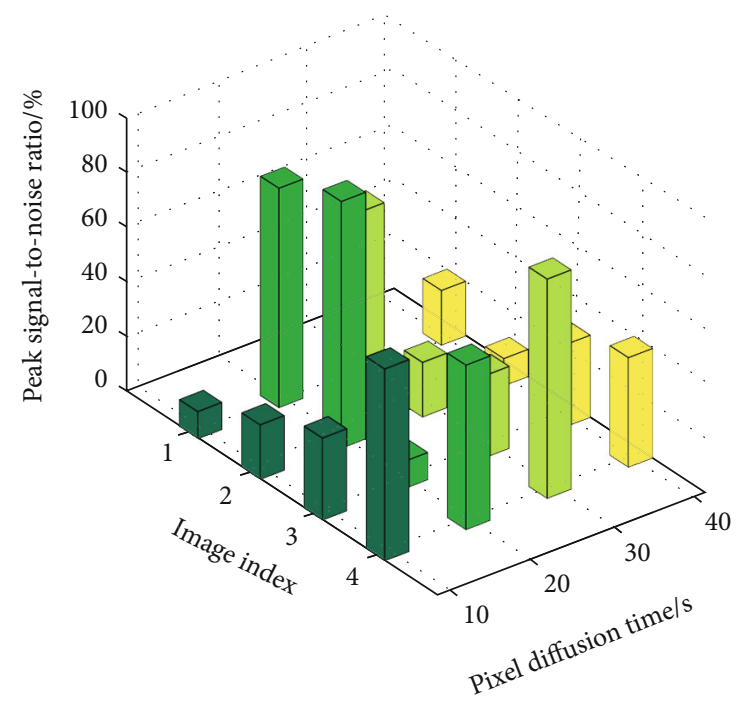

FIGURE 9: Three-dimensional histogram of peak signal-to-noise ratio with pixel diffusion time.

must be reduced. Only in this way can the details and texture information be better preserved, so the peak signal-to-noise ratio depends on pixel diffusion time to decrease.

In order to verify the effect of the partial differential equation algorithm, in the environment shown above, the genetic algorithm, the Voronoi diagram search, the artificial potential field algorithm, the RRT algorithm, and the partial differential equation algorithm in this paper are compared, respectively, to carry out urban route planning. Figure 10 shows a fan chart of comparison of the effects of urban planning image enhancement algorithms based on partial differential equations. Compared with other planning algorithms, the planning algorithm in this paper reduces the planned path length by $33.45 \%, 27.23 \%, 18.21 \%, 15.24 \%$, and $5.87 \%$, respectively. It can be clearly seen that the denoising image of the model proposed in this chapter is clearer,
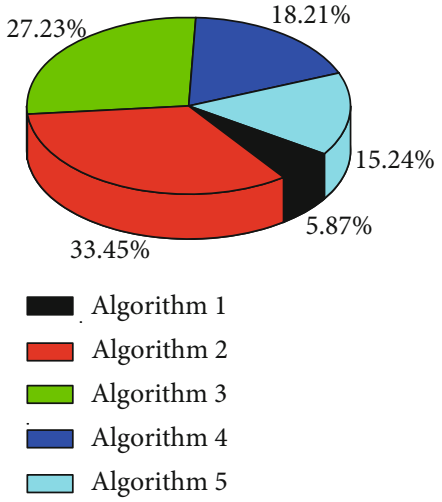

Figure 10: Comparison of the effects of urban planning image enhancement algorithms based on partial differential equations.

namely, it has a good visual effect, indicating that the algorithm can effectively remove noise. In addition, the edge and texture details of the denoised image are also clearer, indicating that the algorithm proposed in this chapter can better preserve the edge and texture information to a certain extent without being destroyed, and more importantly, it reduces the "staircase effect" to a certain extent.

\section{Conclusion}

This paper analyzes the feasibility of the partial differential equation algorithm in urban path planning and proposes a new method of urban path planning. We use partial differential equations to model urban path planning, through the adaptive mesh refinement of the experimental environment; it realizes the application of partial differential equations in urban path planning. Theoretical research shows that it is feasible to solve the path planning of mobile robots by partial differential equation algorithms, which provides new ideas and methods for urban path planning research for static and dynamic. The experimental results prove the effectiveness of the partial differential equation algorithm to solve the urban path planning problem.

The main improvements in this paper are as follows: (1) considering that PM will have a step effect in the process of restoring images, the improved coupling median filter and PM model are proposed with the complex diffusion model. The improved algorithm effectively combines the advantages of various models and improves the visual effect of the image without affecting the denoising performance. (2) The model is unable to effectively remove the shot noise points, so it is improved. A time-weighted selective diffusion model is proposed. The improved algorithm is relative in terms of recovery effect and convergence speed. The model has been improved to some extent. (3) Considering that the total variation model will have a step effect in the process of restoring the image, an improved model that couples the second-order and fourth-order partial differential equations is proposed. The improved algorithm improves the visual effect of the image, and the convergence speed is much higher than that of the fourth-order model. 


\section{Data Availability}

The data used to support the findings of this study are available from the corresponding author upon request.

\section{Conflicts of Interest}

The author declares no known competing financial interests or personal relationships that could have appeared to influence the work reported in this paper.

\section{Acknowledgments}

This study was supported by the Department of Science and Technology Research Project: Theory and Practice of Seismic Optimal Design of Urban and Rural Water Supply Network (Grant No. 202102310246), Henan Provincial Key Scientific Research Projects of Colleges and Universities: Research on Safety Performance and Optimization Design of Urban and Rural Water Supply Network (Grant No. 20A560017), and Henan Province Project Plan of Archival Science and Technology: Study of Earthquake Disaster Emergency Management Mechanism of Archives Department in Henan Province (Grant No. 2021-R-20).

\section{References}

[1] A. Bueno-Orovio, V. M. Perez-Garcia, and F. H. Fenton, "Spectral methods for partial differential equations in irregular domains: the spectral smoothed boundary method," SIAM Journal on Scientific Computing, vol. 28, no. 3, pp. 886-900, 2006.

[2] M. Bertalmío, L.-T. Cheng, S. Osher, and G. Sapiro, "Variational problems and partial differential equations on implicit surfaces," Journal of Computational Physics, vol. 174, no. 2, pp. 759-780, 2021.

[3] E. Burman, S. Claus, P. Hansbo, M. G. Larson, and A. Massing, "CutFEM: discretizing geometry and partial differential equations," International Journal for Numerical Methods in Engineering, vol. 104, no. 7, pp. 472-501, 2019.

[4] J. Sirignano and K. Spiliopoulos, "DGM: a deep learning algorithm for solving partial differential equations," Journal of Computational Physics, vol. 375, pp. 1339-1364, 2018.

[5] M. Raissi, P. Perdikaris, and G. E. Karniadakis, "Physicsinformed neural networks: a deep learning framework for solving forward and inverse problems involving nonlinear partial differential equations," Journal of Computational Physics, vol. 378, pp. 686-707, 2019.

[6] W. L. Oberkampf, S. M. DeLand, B. M. Rutherford, K. V. Diegert, and K. F. Alvin, "Error and uncertainty in modeling and simulation," Reliability Engineering \& System Safety, vol. 75, no. 3, pp. 333-357, 2018.

[7] D. Gaston, C. Newman, G. Hansen, and D. Lebrun-Grandie, "MOOSE: a parallel computational framework for coupled systems of nonlinear equations," Nuclear Engineering and Design, vol. 239, no. 10, pp. 1768-1778, 2019.

[8] W. Lu, M. L. Chen, G. H. Olivera, K. J. Ruchala, and T. R. Mackie, "Fast free-form deformable registration via calculus of variations," Physics in Medicine \& Biology, vol. 49, no. 14, p. 3067, 2019.
[9] Y. Yu and S. T. Acton, "Speckle reducing anisotropic diffusion," IEEE Transactions on Image Processing, vol. 11, no. 11, pp. 1260-1270, 2002.

[10] G. S. Settles and E. E. Covert, "Schlieren and shadowgraph techniques: visualizing phenomena in transport media," Applied Mechanics Reviews, vol. 55, no. 4, pp. B76-B77, 2019.

[11] H. G. Matthies and A. Keese, "Galerkin methods for linear and nonlinear elliptic stochastic partial differential equations," Computer Methods in Applied Mechanics and Engineering, vol. 194, no. 12-16, pp. 1295-1331, 2005.

[12] S. Torquato and H. W. Haslach Jr., "Random heterogeneous materials: microstructure and macroscopic properties," Applied Mechanics Reviews, vol. 55, no. 4, pp. B62-B63, 2020.

[13] Z. Naghavi, A. Moheb, and S. Ziaei-Rad, "Numerical simulation of rough rice drying in a deep-bed dryer using nonequilibrium model," Energy Conversion and Management, vol. 51, no. 2, pp. 258-264, 2018.

[14] L. C. Evans, "Partial differential equations and MongeKantorovich mass transfer," Current Developments in Mathematics, vol. 1997, no. 1, pp. 65-126, 2017.

[15] M. J. Berger and J. Oliger, "Adaptive mesh refinement for hyperbolic partial differential equations," Journal of Computational Physics, vol. 53, no. 3, pp. 484-512, 2019.

[16] D. K. Pai, "Strands: interactive simulation of thin solids using Cosserat models," Computer Graphics Forum, vol. 21, no. 3, pp. 347-352, 2020.

[17] C. H. Huo and L. H. Chai, "Physical principles and simulations on the structural evolution of eco-industrial systems," Journal of Cleaner Production, vol. 16, no. 18, pp. 1995-2005, 2018.

[18] D. Helbing, A. Hennecke, V. Shvetsov, and M. Treiber, "MASTER: macroscopic traffic simulation based on a gas-kinetic, non-local traffic model," Transportation Research Part B: Methodological, vol. 35, no. 2, pp. 183-211, 2021.

[19] F. Lindgren, H. Rue, and J. Lindström, "An explicit link between Gaussian fields and Gaussian Markov random fields: the stochastic partial differential equation approach," Journal of the Royal Statistical Society: Series B (Statistical Methodology), vol. 73, no. 4, pp. 423-498, 2011.

[20] G. Rozza, D. B. P. Huynh, and A. T. Patera, "Reduced basis approximation and a posteriori error estimation for affinely parametrized elliptic coercive partial differential equations," Archives of Computational Methods in Engineering, vol. 15, no. 3, 2007.

[21] C. Ronchi, R. Iacono, and P. S. Paolucci, "The "cubed sphere": a new method for the solution of partial differential equations in spherical geometry," Journal of Computational Physics, vol. 124, no. 1, pp. 93-114, 1996. 